\title{
A new perspective on independent component analysis
}

\author{
H. Zhao*1 and M. Grigoriu ${ }^{\dagger 1}$ \\ ${ }^{1}$ School of Civil and Environmental Engineering, Cornell University, USA
}

\begin{abstract}
The paper examines the performance of the independent component analysis(ICA) which represents random vectors $\mathbf{X}$ by vectors $\tilde{\mathbf{X}}$ whose components are linear forms of independent random variables. The representation holds exactly or asymptotically in tails for Gaussian and non-Gaussian vectors with special characteristic functions or Gaussian, translation, and non-Gaussian vectors with independent tails. However, the distributions of $\tilde{\mathbf{X}}$ and its target vector $\mathbf{X}$ differ for a board range of non-Gaussian vectors. This statement is supported by numerical examples and theoretical arguments. It is also shown that the ICA representation $\tilde{\mathbf{X}}$ is approximately Gaussian for high dimensional vectors.
\end{abstract}

Keywords: Independent component analysis; Gaussian and non-Gaussian vectors; Translation vectors; Tail independent vectors; Wind engineering.

\section{Introduction}

Independent component analysis(ICA) is an useful tool in data processing and has been used successfully in several fields, e.g., image recognition [6], market forecast [1], and wind engineering [3]. In ICA, a random vector is represented by a linear combination of deterministic vectors with the same dimension, referred to as modes, modulated by independent random variables. This representation has two benefits. First, it provides an efficient algorithm for generating samples

*hz289@cornell.edu

${ }^{\dagger}$ mdg12@cornell.edu 
of dependent random variables from samples of independent variables. Second, the modes have various meaningful interpretations in different applications. For example, the ICA modes for the wind-induced pressure field on bluff bodies have the meaning of so-called coherent structures which reveal the flow behavior, detect the recurrent appearances of pressure patterns, and provide the information of vortex shedding in the neighborhood of the body [3].

Let $\mathbf{X} \in \mathbb{R}^{m}$ be an $m$-dimensional random vector with covariance matrix $\boldsymbol{\Sigma}$ and joint distribution F. It is assumed without loss of generality that $\mathbf{X}$ has mean zero. The ICA represents $\mathbf{X}$ by $\tilde{\mathbf{X}}$ which has the form

$$
\mathbf{X} \approx \tilde{\mathbf{X}}=\mathbf{A} \mathbf{S}=\sum_{k=1}^{n} A_{k} S_{k}
$$

where $\mathbf{S}=\left[S_{1}, \ldots, S_{k}, \ldots, S_{n}\right]^{T}$ is an $n$-dimensional vector with independent components $\left\{S_{k}\right\}(n \leq$ $m)$, and $\mathbf{A}=\left[A_{1}, \ldots, A_{k}, \ldots, A_{n}\right]$ is an $m \times n$ deterministic matrix, where the columns $A_{k} \in \mathbb{R}^{m}$, $k=1, \ldots, n$, are modes [3].

We show that (1) it may not be possible to represent $\mathbf{X}$ by $\tilde{\mathbf{X}}$ exactly, i.e., $\mathbf{X} \stackrel{\mathrm{d}}{=} \tilde{\mathbf{X}}$ does not, generally, hold if $\left\{S_{k}\right\}$ are independent, and (2) the discrepancy between the target vector $\mathbf{X}$ and its ICA representation $\tilde{\mathbf{X}}$ can be significant. We only consider the case $n=m$. The construction of $\tilde{\mathbf{X}}$ for this case involves three steps. First, denote by $L$ the Cholesky lower triangular matrix of known correlation matrix $\boldsymbol{\Sigma}$ of $\mathbf{X}$. Matrix $L$ relates $\boldsymbol{\Sigma}$ through equation $\boldsymbol{\Sigma}=L L^{T}$, whose entries $\left\{L_{j k}\right\}$ can be calculated by $[9$, Sec. 5.2 .1$]$

$$
L_{j k}=\frac{\Sigma_{j k}-\sum_{r=1}^{k-1} L_{j r} L_{k r}}{\left[\Sigma_{k k}-\sum_{r=1}^{k-1} L_{k r}^{2}\right]^{1 / 2}}, \quad 1 \leq k \leq j \leq m, \text { with } \sum_{r=1}^{0} L_{j r} L_{k r}=0
$$

where $\left\{\Sigma_{j k}\right\}$ are the components of $\boldsymbol{\Sigma}$. Second, set $\mathbf{A}=L \Lambda$, where $\Lambda$ is an arbitrary orthogonal matrix [4]. Note that $E[\tilde{\mathbf{X}}]=E[\mathbf{X}]=\mathbf{0}$ and

$$
E\left[\tilde{\mathbf{X}} \tilde{\mathbf{X}}^{T}\right]=E\left[\mathbf{A S S}^{T} \mathbf{A}^{T}\right]=L \Lambda E\left[\mathbf{S S}^{T}\right] \Lambda^{T} L^{T}=L \Lambda \Lambda^{T} L^{T}=L L^{T}=\boldsymbol{\Sigma}=E\left[\mathbf{X} \mathbf{X}^{T}\right], \forall \Lambda
$$

provided $E[\mathbf{S}]=\mathbf{0}$ and $E\left[\mathbf{S S}^{T}\right]=\mathbf{I}$, in which $\mathbf{0}$ is a zero vector and $\mathbf{I}$ is the identity matrix. Third, we use a metric to quantify the degree of dependence among the components of $\mathbf{S}=\Lambda^{T} L^{-1} \mathbf{X}$ to 
find an optimal $\Lambda$, e.g., the metric $d(\mathbf{S})=\left|\sum_{k=1}^{m} H\left(S_{k}\right)-H(\mathbf{S})\right|$, where $H\left(S_{k}\right)$ and $H(\mathbf{S})$ are entropies defined by $H\left(S_{k}\right)=-\int f_{S_{k}} \log f_{S_{k}} d s_{k}$ and $H(\mathbf{S})=-\int f_{\mathbf{S}} \log f_{\mathbf{S}} d s_{1} \ldots d s_{m}$, respectively, in which $f_{S_{k}}$ and $f_{\mathbf{S}}$ are marginal and joint densities of $\mathbf{S}[5,11]$. Note that $d(\mathbf{S})=0$ iff $\mathbf{S}$ has independent components. Alternative metrics can be found in $[14,2,17]$.

This paper has two parts. The first part presents cases in which the ICA representation $\tilde{\mathbf{X}}$ fully or partially characterizes $\mathbf{X}$. The second part provides several examples in which $\tilde{\mathbf{X}}$ and $\mathbf{X}$ differ and examines the accuracy of $\tilde{\mathbf{X}}$ for low and high dimensional vectors.

\section{Exact ICA representation}

We present two classes of random vectors that admit the representation in Eq. 1, Gaussian vectors and non-Gaussian vectors whose characteristic functions satisfy some conditions, and a class of non-Gaussian vectors for which Eq. 1 holds asymptotically in tails.

\subsection{Gaussian vectors}

If $\mathbf{X} \in \mathbb{R}^{m}$ is an $m$-dimensional Gaussian random vector with mean zero and correlation matrix $\mathbf{\Sigma}$, then

$$
\mathbf{X} \stackrel{\mathrm{d}}{=} \tilde{\mathbf{X}}=L \mathbf{S}
$$

where $L$ is the Cholesky lower triangular matrix of $\boldsymbol{\Sigma}$ and $\left\{S_{k}\right\}$, the components of $\mathbf{S}$, are independent and identically standard Gaussian variables. The proof of the validity of Eq. 4 can be found in [16, Sec. 3.5.3].

\subsection{Non-Gaussian vectors}

The distributions of $\tilde{\mathbf{X}}$ and $\mathbf{X}$ are identical iff there exists an $\mathbf{A}$ such that the characteristic function of $\mathbf{X}$ satisfies the following condition

$$
\begin{aligned}
\varphi_{X}\left(\mathbf{W}^{T} \mathbf{v}\right)=E\left[\exp \left\{i \mathbf{v}^{T} \mathbf{W} \mathbf{X}\right\}\right] & =\prod_{k=1}^{m} E\left[\exp \left\{i v_{k} S_{k}\right\}\right]=\prod_{k=1}^{m} E\left[\exp \left\{i \sum_{j=1}^{m} W_{k j} v_{k} X_{j}\right\}\right] \\
& =\prod_{k=1}^{m} \varphi_{X}\left(W_{k 1} v_{k}, \ldots, W_{k j} v_{k}, \ldots, W_{k m} v_{k}\right),
\end{aligned}
$$


where $\left\{S_{k}\right\},\left\{X_{j}\right\}$, and $\left\{W_{k j}\right\}$ are components of $\mathbf{S}, \mathbf{X}$, and $\mathbf{W}=\mathbf{A}^{-1}$, respectively. This follows from the definition of $\tilde{\mathbf{X}}$ and the property of characteristic functions for independent random variables.

It is possible to satisfy the condition in Eq. 5 . For example, Eq. 5 becomes

$$
\varphi_{X}\left(W_{11} v_{1}+W_{21} v_{2}, W_{12} v_{1}+W_{22} v_{2}\right)=\varphi_{X}\left(W_{11} v_{1}, W_{12} v_{1}\right) \varphi_{X}\left(W_{21} v_{2}, W_{22} v_{2}\right)
$$

for $m=2$ and holds for a bivariate uniform vector $\mathbf{X}=\left[X_{1}, X_{2}\right]^{T}$ with probability density as shown in Fig. 1. The density and characteristic function of $\mathbf{X}$ in this figure are

$$
\begin{aligned}
f\left(x_{1}, x_{2}\right) & = \begin{cases}\frac{1}{4}, & \text { for } x_{1}, x_{2} \in A_{u 1} . \\
0, & \text { otherwise. }\end{cases} \\
\text { and } \varphi\left(v_{1}, v_{2}\right) & =\frac{2\left[\cos \left(v_{1}\right)-\cos \left(2 v_{2}\right)\right]}{4 v_{2}^{2}-v_{1}^{2}},
\end{aligned}
$$

respectively. Note that Eq. 6 holds when $W_{11}=2 W_{12}$ and $W_{21}=2 W_{22}$ so that there exists such a matrix $\mathbf{A}$ that $\tilde{\mathbf{X}} \stackrel{\mathrm{d}}{=} \mathbf{X}$.

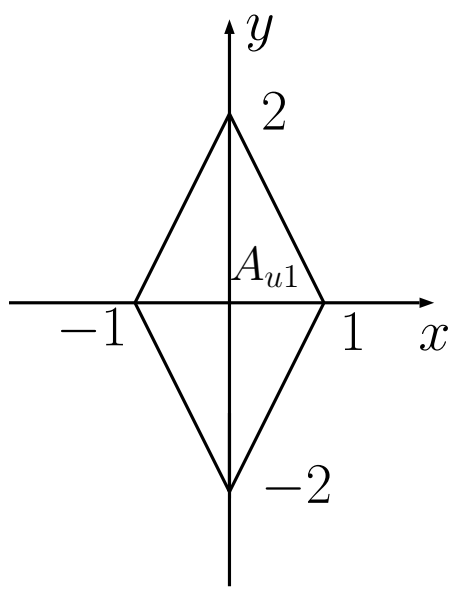

Figure 1: PDF of bivariate uniform distribution

\subsection{Vectors with asymptotically independent components}

Consider a special case of $\tilde{\mathbf{X}}$ by setting $\mathbf{A}=\mathbf{I}$. Then, Eq. 1 becomes

$$
\tilde{\mathbf{X}} \stackrel{\mathrm{d}}{=} \mathbf{S}
$$


where $\mathbf{S}$ has independent components $\left\{S_{j}\right\}$ which satisfy the condition $S_{j} \stackrel{\mathrm{d}}{=} X_{j}$, and so does $\tilde{\mathbf{X}}$. Denote by $\tilde{X}_{j}, j=1, \ldots, m$, the components of $\tilde{\mathbf{X}}$. We show that $\tilde{\mathbf{X}}$ and $\mathbf{X}$ have similar tails, i.e.,

$$
\lim _{x_{1}, \ldots, x_{m} \rightarrow \infty}\left[P\left(\bigcap_{j=1}^{m}\left\{X_{j} \leq x_{j}\right\}\right)-\prod_{j=1}^{m} P\left(\tilde{X}_{j} \leq x_{j}\right)\right]=0
$$

if $\mathbf{X}$ is Gaussian or translation with dependent components, and if $\mathbf{X}$ is a non-Gaussian, nontranslation vector with independent tails.

\subsubsection{Gaussian vectors}

Suppose $\mathbf{X}$ is a standard Gaussian random vector. The proof of Eq. 10 follows from (1) the fact that $X_{j}$ and $\tilde{X}_{j}$ have the same distributions, i.e., $X_{j} \stackrel{\mathrm{d}}{=} \tilde{X}_{j}, j=1, \ldots, m,(2)$ Corollary to the Normal Comparison Lemma which states that [13, Corollary 2.1]

$$
\left|P\left(\bigcap_{j=1}^{m}\left\{X_{j} \leq x_{j}\right\}\right)-\prod_{j=1}^{m} P\left(X_{j} \leq x_{j}\right)\right| \leq \frac{1}{4} \sum_{1 \leq r<t \leq m}\left|\rho_{r t}\right| \exp \left[-\frac{x_{r}^{2}+x_{t}^{2}}{2\left(1+\left|\rho_{r t}\right|\right)}\right],
$$

where $\rho_{r t}$ is the covariance between $X_{r}$ and $X_{t}$, and (3) the observation that the upper bound in Eq. 11 approaches zero as $x_{1}, \ldots, x_{m} \rightarrow \infty$.

Consider for illustration a bivariate Gaussian vector with mean zero and correlation matrix $\boldsymbol{\Sigma}$. The components of $\boldsymbol{\Sigma}$ are $\Sigma_{11}=\Sigma_{22}=1$ and $\Sigma_{12}=\Sigma_{21}=0.5$. Fig. 2 shows the probabilities $1-P\left(X_{1} \leq x, X_{2} \leq x\right)$ and $1-P\left(\tilde{X}_{1} \leq x\right) P\left(\tilde{X}_{2} \leq x\right)$. It can be seen that $P\left(X_{1} \leq x, X_{2} \leq x\right) \approx$ $P\left(\tilde{X}_{1} \leq x\right) P\left(\tilde{X}_{2} \leq x\right)$ for sufficiently large $x$.

\subsubsection{Translation vectors}

Let $X_{j}=h_{j}\left(G_{j}\right), j=1, \ldots, m$, denote the components of a translation vector, where $\left\{G_{j}\right\}$ are correlated standard Gaussian variables and $h_{j}=F_{j}^{-1} \circ \Phi$ are generally nonlinear functions, in which $F_{j}$ and $\Phi$ denote the distribution of $X_{j}$ and standard Gaussian distribution, respectively [8, Sec. 3.1]. Note that Eq. 10 can be written as

$$
\lim _{u_{1}, \ldots, u_{m} \rightarrow 1}\left[P\left(\bigcap_{j=1}^{m}\left\{X_{j} \leq F_{j}^{-1}\left(u_{j}\right)\right\}\right)-\prod_{j=1}^{m} u_{j}\right]=0
$$



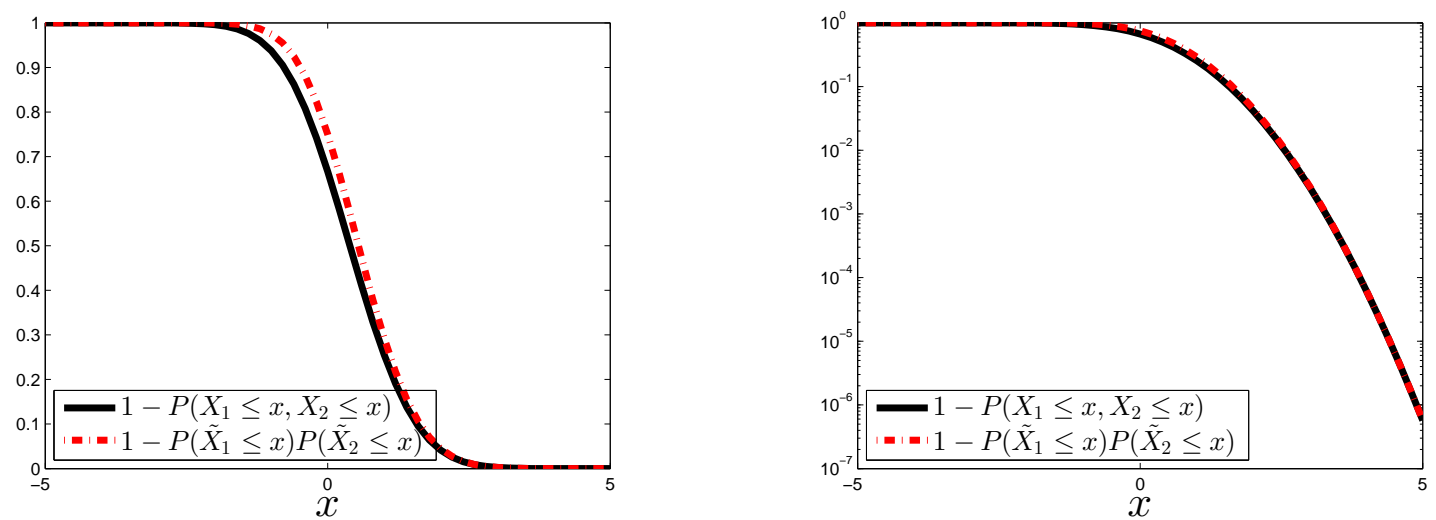

Figure 2: Plots of $1-P\left(X_{1} \leq x, X_{2} \leq x\right)$ and $1-P\left(\tilde{X}_{1} \leq x\right) P\left(\tilde{X}_{2} \leq x\right)$ for bivariate standard Gaussian vector with $\rho=0.5$ : linear (a) and logarithmic (b) scales

since $P\left(\tilde{X}_{j} \leq F_{j}^{-1}\left(u_{j}\right)\right)=u_{j}, j=1, \ldots, m$.

The limit in Eq. 12 holds by the definition of translation vectors and the property of Gaussian vectors. We have

$$
\begin{aligned}
\lim _{u_{1}, \ldots, u_{m} \rightarrow 1} P\left(\bigcap_{j=1}^{m}\left\{X_{j} \leq F_{j}^{-1}\left(u_{j}\right)\right\}\right) & =\lim _{u_{1}, \ldots, u_{m} \rightarrow 1} P\left(\bigcap_{j=1}^{m}\left\{F_{j}^{-1}\left(\Phi\left(G_{j}\right)\right) \leq F_{j}^{-1}\left(u_{j}\right)\right\}\right) \\
& =\lim _{u_{1}, \ldots, u_{m} \rightarrow 1} P\left(\bigcap_{j=1}^{m}\left\{G_{j} \leq \Phi^{-1}\left(u_{j}\right)\right\}\right) \\
& =\lim _{u_{1}, \ldots, u_{m} \rightarrow 1} \prod_{j=1}^{m} u_{j} .
\end{aligned}
$$

The above property can be illustrated by Fig. 3 which shows $1-P\left(X_{1} \leq x, X_{2} \leq x\right)$ and $1-P\left(\tilde{X}_{1} \leq x\right) P\left(\tilde{X}_{2} \leq x\right)$ for a bivariate translation vector with standard exponential marginal distributions $F_{1}(x)=F_{2}(x)=1-\exp (-x)$. The probabilities $P\left(X_{1} \leq x, X_{2} \leq x\right)$ and $P\left(\tilde{X}_{1} \leq\right.$ $x) P\left(\tilde{X}_{2} \leq x\right)$ match in the tail region.

\subsubsection{Non-Gaussian, non-translation vectors with independent tails}

Consider a bivariate random vector $\mathbf{X}$ with components $X_{1}$ and $X_{2}$ with distributions $F_{1}$ and $F_{2}$, respectively. Define by $\lambda_{u}=P\left(X_{2}>F_{2}^{-1}(u) \mid X_{1}>F_{1}^{-1}(u)\right)$ the upper tail dependence coefficient. If

$$
\lim _{u \rightarrow 1} \lambda_{u}=0,
$$



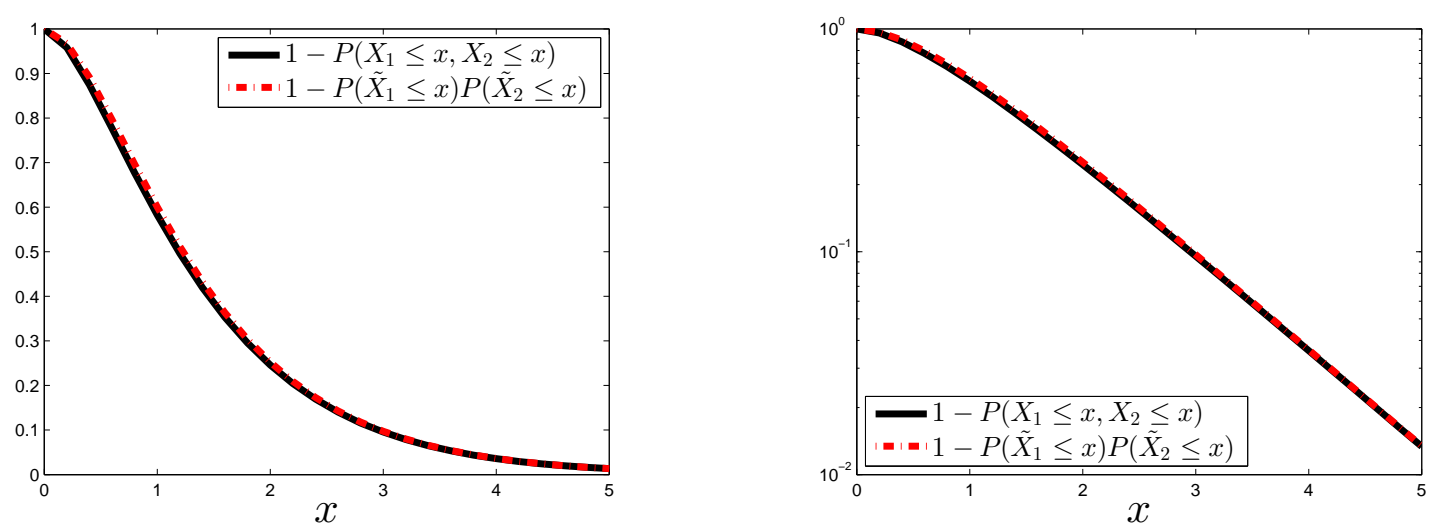

Figure 3: Plots of $1-P\left(X_{1} \leq x, X_{2} \leq x\right)$ and $1-P\left(\tilde{X}_{1} \leq x\right) P\left(\tilde{X}_{2} \leq x\right)$ for bivariate translation vector with $F_{1}(x)=F_{2}(x)=1-\exp (-x)$ : linear (a) and logarithmic (b) scales

then we say that $\mathbf{X}$ is upper tail independent [10, Sec. 2.1.10]. We show that this definition implies that $P\left(X_{1} \leq F_{1}^{-1}(u), X_{2} \leq F_{2}^{-1}(u)\right)$ approaches $P\left(X_{1} \leq F_{1}^{-1}(u)\right) P\left(X_{2} \leq F_{2}^{-1}(u)\right)$ for $u$ arbitrarily close to 1, in other words, Eq. 14 is a sufficient condition for

$$
\lim _{u \rightarrow 1}\left[P\left(X_{1} \leq F_{1}^{-1}(u), X_{2} \leq F_{2}^{-1}(u)\right)-u^{2}\right]=0 .
$$

If Eq. 14 holds, then

$$
\begin{aligned}
0 & =\lim _{u \rightarrow 1} \frac{P\left(X_{1}>F_{1}^{-1}(u), X_{2}>F_{2}^{-1}(u)\right)}{P\left(X_{1}>F_{1}^{-1}(u)\right)} \\
& =\lim _{u \rightarrow 1} \frac{1-P\left(X_{1} \leq F_{1}^{-1}(u)\right)-P\left(X_{2} \leq F_{2}^{-1}(u)\right)+P\left(X_{1} \leq F_{1}^{-1}(u), X_{2} \leq F_{2}^{-1}(u)\right)}{1-P\left(X_{1} \leq F_{1}^{-1}(u)\right)} \\
& =\lim _{u \rightarrow 1} \frac{1-2 u+P\left(X_{1} \leq F_{1}^{-1}(u), X_{2} \leq F_{2}^{-1}(u)\right)}{1-u} \\
& =2-\lim _{u \rightarrow 1} \frac{P\left(X_{1} \leq F_{1}^{-1}(u), X_{2} \leq F_{2}^{-1}(u)\right)-1}{u-1} \times \\
& \lim _{u \rightarrow 1} \frac{\log u}{\log P\left(X_{1} \leq F_{1}^{-1}(u), X_{2} \leq F_{2}^{-1}(u)\right)} \frac{\log P\left(X_{1} \leq F_{1}^{-1}(u), X_{2} \leq F_{2}^{-1}(u)\right)}{\log u} \\
& =2-\lim _{u \rightarrow 1} \frac{\log P\left(X_{1} \leq F_{1}^{-1}(u), X_{2} \leq F_{2}^{-1}(u)\right)}{\log u}
\end{aligned}
$$

Let $f(u)$ denote $\log u / \log P\left(X_{1} \leq F_{1}^{-1}(u), X_{2} \leq F_{2}^{-1}(u)\right)$. Note that Eq. 16 holds since

$$
\lim _{u \rightarrow 1} f(u)=\lim _{u \rightarrow 1} \frac{P\left(X_{1} \leq F_{1}^{-1}(u), X_{2} \leq F_{2}^{-1}(u)\right)}{u \frac{d}{d u} P\left(X_{1} \leq F_{1}^{-1}(u), X_{2} \leq F_{2}^{-1}(u)\right)}=\frac{1}{2}<\infty
$$


Then, by multiplying $\lim _{u \rightarrow 1} \log u$ on both sides of Eq. 16, we have

$$
\begin{aligned}
0 & =\lim _{u \rightarrow 1} \frac{\log P\left(X_{1} \leq F_{1}^{-1}(u), X_{2} \leq F_{2}^{-1}(u)\right)}{\log u} \lim _{u \rightarrow 1} \log u-2 \lim _{u \rightarrow 1} \log u \\
& =\lim _{u \rightarrow 1}\left[P\left(X_{1} \leq F_{1}^{-1}(u), X_{2} \leq F_{2}^{-1}(u)\right)-u^{2}\right] .
\end{aligned}
$$

On the other hand, Eq. 15 does not imply Eq. 14. One counterexample is a bivariate vector with distribution

$$
\begin{gathered}
F\left(x_{1}, x_{2}\right)=\exp \left\{-\left[x_{1}^{-\frac{1}{\theta}}+x_{2}^{-\frac{1}{\theta}}\right]^{\theta}\right\} \\
x_{1}, x_{2} \geq 0, \quad \theta \in(0,1] .
\end{gathered}
$$

This distribution satisfies Eq. 15 but $\lambda_{u}=2-2^{\theta} \neq 0$ if $\theta \neq 1$.
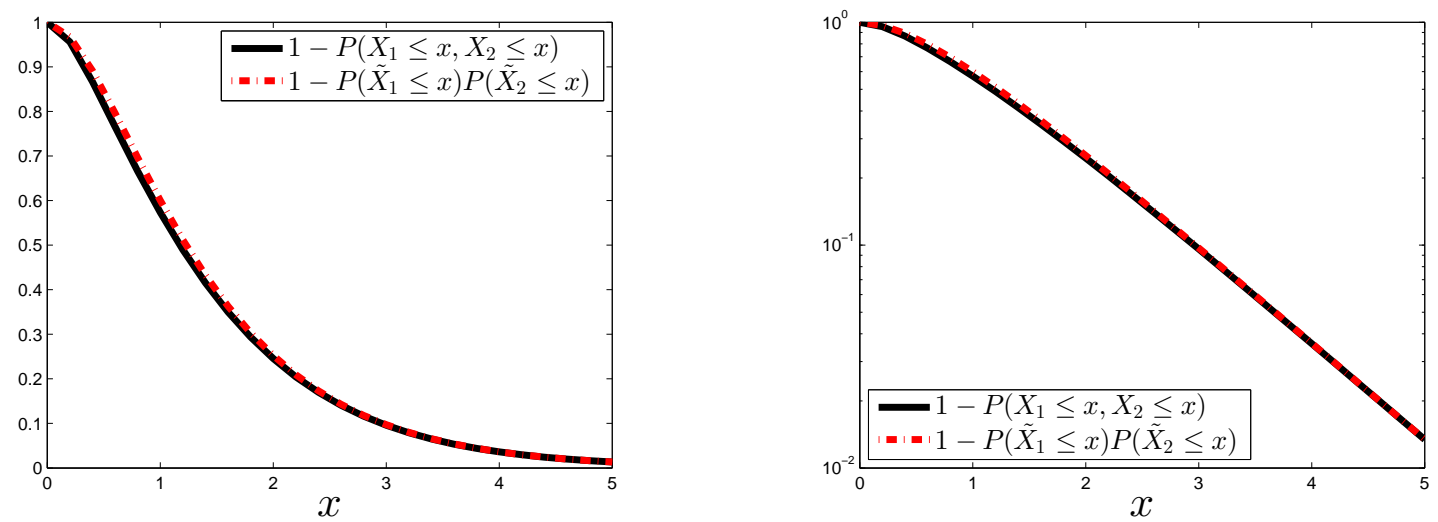

Figure 4: Plots of $1-P\left(X_{1} \leq x, X_{2} \leq x\right)$ and $1-P\left(\tilde{X}_{1} \leq x\right) P\left(\tilde{X}_{2} \leq x\right)$ for bivariate Gumbel distribution with $\alpha=0.5$ : linear (a) and logarithmic (b) scales

An example for upper tail independent random vector is a bivariate Gumbel vector with joint and marginal distributions of $X_{1}$ and $X_{2}$ defined by [12, Sec. 2.2]

$$
\begin{gathered}
F\left(x_{1}, x_{2}\right)=\left(1-\exp \left(-x_{1}\right)\right)\left(1-\exp \left(-x_{2}\right)\right)\left(1+\alpha \exp \left(-x_{1}-x_{2}\right)\right) \\
x_{1}, x_{2} \geq 0, \quad \alpha \in[-1,1]
\end{gathered}
$$

and

$$
F_{j}\left(x_{j}\right)=1-\exp \left(-x_{j}\right), \quad j=1,2,
$$


respectively. Note that this is not a translation vector since $f\left(x_{1}, x_{2}\right) \prod_{j=1}^{2} \phi\left(g_{j}\right) / f\left(x_{j}\right)$ does not admit the form of Gaussian joint density with arguments $g_{j}, j=1,2$, where $g_{j}=\Phi^{-1}\left(F_{j}\left(x_{j}\right)\right)$, $\phi$ is standard Gaussian density, and $f\left(x_{1}, x_{2}\right)$ and $f\left(x_{j}\right), j=1,2$, denote the joint and marginal densities of $X_{1}$ and $X_{2}$, respectively. Fig. 4 shows the probabilities $1-P\left(X_{1} \leq x, X_{2} \leq x\right)$ and $1-P\left(\tilde{X}_{1} \leq x\right) P\left(\tilde{X}_{2} \leq x\right)$ with $\alpha=0.5$. It can be seen that $P\left(X_{1} \leq x, X_{2} \leq x\right) \approx P\left(\tilde{X}_{1} \leq\right.$ $x) P\left(\tilde{X}_{2} \leq x\right)$ in the tail region which supports Eq. 15 .

\section{Approximate ICA representation}

We give examples of low dimensional non-Gaussian vectors for which the ICA representation does not hold, and examine the accuracy of $\tilde{\mathbf{X}}$ for this case. We also show that the ICA representation $\tilde{\mathbf{X}}$ for arbitrary high dimensional non-Gaussian vectors is approximately Gaussian.

\subsection{Low dimensional vectors}

There are numerous non-Gaussian vectors that do not admit the ICA representation. We give one example to support this statement. The components $X_{1}$ and $X_{2}$ of $\mathbf{X}$ have joint density $f\left(x_{1}, x_{2}\right)=(2 \pi)^{-1}\left(1+x_{1}^{2}+x_{2}^{2}\right)^{-\frac{3}{2}}$, which is known as the circular bivariate Cauchy distribution. The characteristic function of $\mathbf{X}$ is $\varphi_{X}\left(v_{1}, v_{2}\right)=\exp \left\{-\left(v_{1}^{2}+v_{2}^{2}\right)^{\frac{1}{2}}\right\}$ [7]. The equality of Eq. 6 holds iff $W_{11} W_{22}+W_{12} W_{21}=0$ which indicates the singularity of matrix $\mathbf{W}=\mathbf{A}^{-1}$. Therefore, matrix A does not exist in this example.
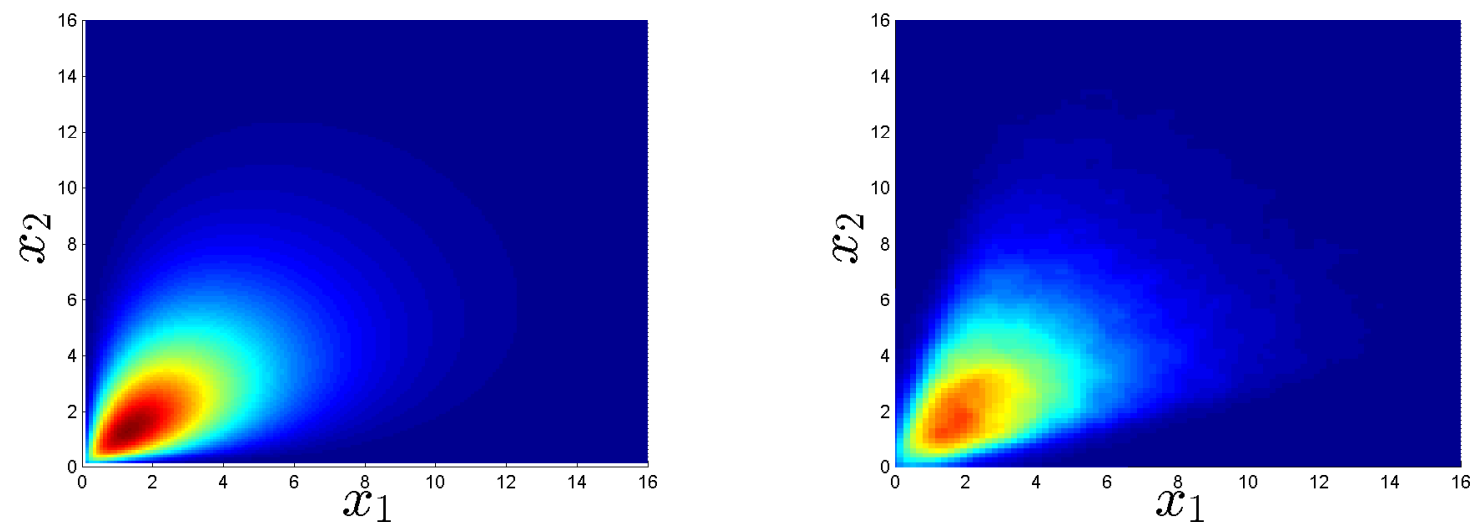

Figure 5: Joint densities of target vector $\mathbf{X}$ (a) and the ICA representation $\tilde{\mathbf{X}}$ (b) 

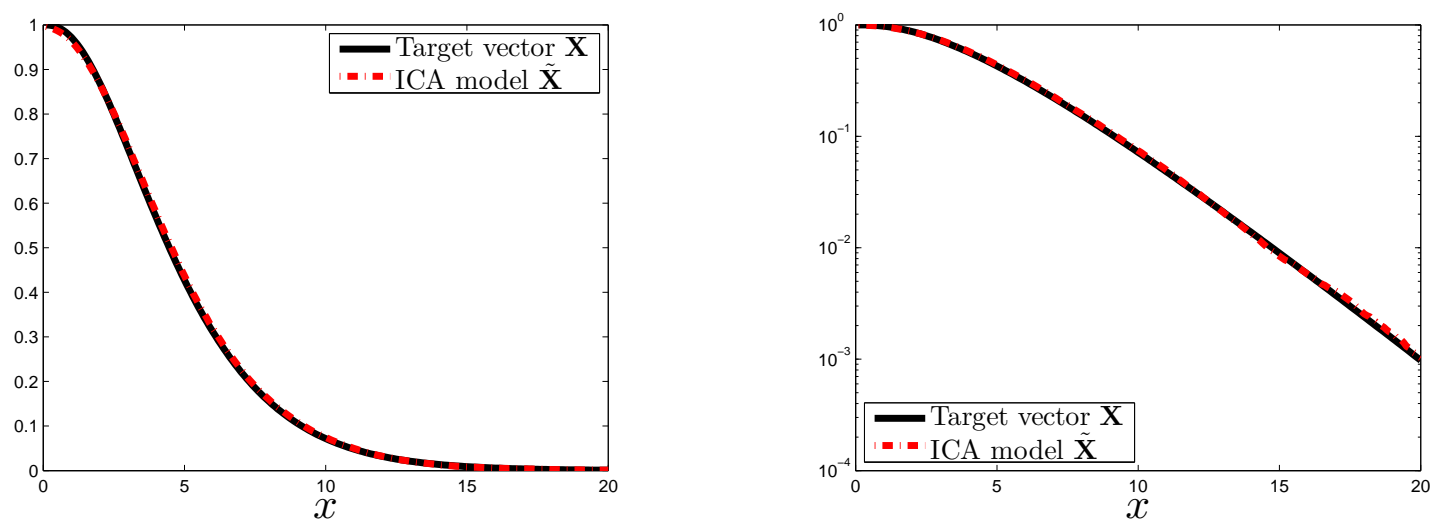

Figure 6: Plots of $1-P\left(X_{1} \leq x, X_{2} \leq x\right)$ and $1-P\left(\tilde{X}_{1} \leq x, \tilde{X}_{2} \leq x\right)$ of $\mathbf{X}$ and $\tilde{\mathbf{X}}$ for bivariate translation vectors with Gamma marginals: linear (a) and logarithmic (b) scales

Moreover, two examples are provided to reveal the performance of $\tilde{\mathbf{X}}$ for low dimensional vectors. The ICA representation $\tilde{\mathbf{X}}$ may be a satisfactory approximation of $\mathbf{X}$. The example for illustration is a bivariate translation vector with components $X_{j}=F_{j}^{-1}\left(\Phi\left(G_{j}\right)\right), j=1,2$, where $\left\{G_{1}, G_{2}\right\}$ are correlated standard Gaussian variables with correlation coefficient $\rho=0.5$, and $F_{1}=F_{2}$ are Gamma distribution with shape coefficient $k=2$ and scale coefficient $\theta=2$. The joint density of $\tilde{\mathbf{X}}$ almost coincides with that of target vector $\mathbf{X}$, as shown in Fig. 5 . Fig. 6 shows the probabilities $1-P\left(X_{1} \leq x, X_{2} \leq x\right)$ and $1-P\left(\tilde{X}_{1} \leq x, \tilde{X}_{2} \leq x\right)$. The differences of two lines are almost indistinguishable at the figure scale. Suppose now $\mathbf{X}=\left[X_{1}, X_{2}\right]^{T}$ is a bivariate zero-mean Tdistributed random vector with density

$$
f\left(x_{1}, x_{2}\right)=\frac{\Gamma[\nu / 2+1]}{\Gamma(\nu / 2) \nu \pi \sqrt{|\mathbf{\Sigma}|}\left[1+\mathbf{x}^{T} \mathbf{\Sigma}^{-1} \mathbf{x} / \nu\right]^{\nu / 2+1}}=\frac{1}{2 \pi \sqrt{|\boldsymbol{\Sigma}|}\left[1+\mathbf{x}^{T} \boldsymbol{\Sigma}^{-1} \mathbf{x} / \nu\right]^{\nu / 2+1}},
$$

where $\mathbf{x}=\left[x_{1}, x_{2}\right]^{T}, \nu>2$ is the degree of freedom, $\Gamma$ denotes Gamma function, and $\boldsymbol{\Sigma}$ is the correlation matrix. Consider the special case where the components of correlation matrix $\Sigma_{i j}=\delta_{i j}$, in which $\delta_{i j}$ indicates the Kronecker delta function. Then, equation $\mathbf{S}=\Lambda^{T} L^{-1} \mathbf{X}$ takes the form

$$
\begin{aligned}
S_{1} & =\frac{1}{a} \cos \psi X_{1}+\frac{1}{a} \sin \psi X_{2}, \\
S_{2} & =-\frac{1}{a} \sin \psi X_{1}+\frac{1}{a} \cos \psi X_{2}
\end{aligned}
$$

where $a=\sqrt{\nu /(\nu-2)}$ and $\Lambda$ has columns $[\cos \psi, \sin \psi]^{T}$ and $[-\sin \psi, \cos \psi]^{T}$. The joint density of 
$\mathbf{S}$, denoted by $f_{\mathbf{S}}\left(s_{1}, s_{2}\right)$, has the expression

$$
\begin{aligned}
f_{\mathbf{S}}\left(s_{1}, s_{2}\right) & =f\left(a \cos \psi s_{1}-a \sin \psi s_{2}, a \sin \psi s_{1}+a \cos \psi s_{2}\right) \\
& =\frac{1}{2 \pi}\left[1+a^{2}\left(s_{1}^{2}+s_{2}^{2}\right) / \nu\right]^{-(\nu / 2+1)}
\end{aligned}
$$

We can see that $f_{\mathbf{S}}\left(s_{1}, s_{2}\right)$ is irrespective of the direction $\psi$ which implies that there is no theoretical optimal $\Lambda$. However, optimal $\Lambda$ results if the information of $\mathbf{X}$ consists of a finite number of samples. Figs. 7 and 8 show the joint densities of $\mathbf{X}$ and $\tilde{\mathbf{X}}$ and the probabilities $1-P\left(X_{1} \leq x, X_{2} \leq x\right)$ and $1-P\left(\tilde{X}_{1} \leq x, \tilde{X}_{2} \leq x\right)$ for $\nu=5$ from 100000 samples. The differences in the tail region are large.
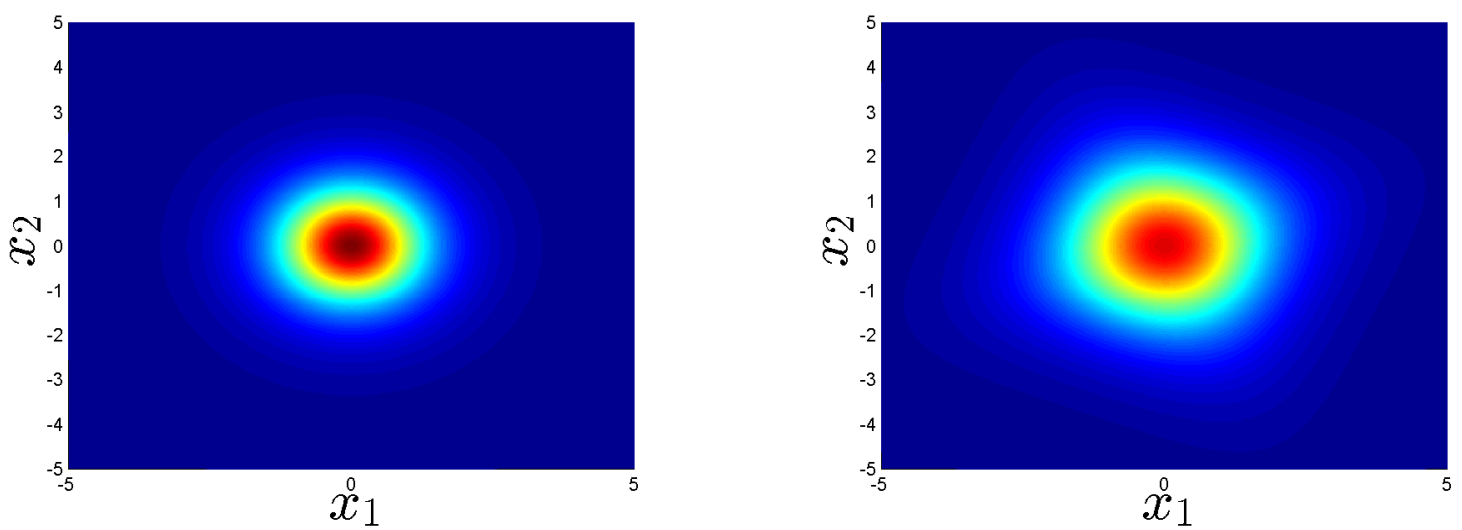

Figure 7: Joint densities of target vector $\mathbf{X}$ (a) and the ICA representation $\tilde{\mathbf{X}}$ (b)


Figure 8: Plots of $1-P\left(X_{1} \leq x, X_{2} \leq x\right)$ and $1-P\left(\tilde{X}_{1} \leq x, \tilde{X}_{2} \leq x\right)$ of $\mathbf{X}$ and $\tilde{\mathbf{X}}$ for bivariate T-distributed vectors: linear (a) and logarithmic (b) scales 


\subsection{High dimensional vectors}

The ICA representation $\tilde{\mathbf{X}}$ performs poorly for high dimensional vectors. We prove this statement by using the Lindeberg-Feller Central Limit Theorem that is stated here for convenience.

Suppose $\left\{S_{k}, k=1, \ldots, n\right\}$ is a set of independent random variables with mean zero, variances $\sigma_{k}^{2}=E\left[S_{k}^{2}\right]$, and distributions $F_{k}^{S}$. Set $w_{n}^{2}=\sum_{k=1}^{n} \sigma_{k}^{2}$ and $W_{n}=S_{1}+\cdots+S_{n}$. We say that $\left\{S_{k}\right\}$ satisfies the Lindeberg-Feller condition if we have

$$
\frac{1}{w_{n}^{2}} \sum_{k=1}^{n} E\left[S_{k}^{2} 1_{\left(\left|S_{k} / w_{n}\right|>\xi\right)}\right]=\frac{1}{w_{n}^{2}} \sum_{k=1}^{n} \int_{\left|s_{k}\right|>\xi w_{n}} s_{k}^{2} F_{k}^{S}\left(d s_{k}\right) \rightarrow 0, \text { as } n \rightarrow \infty, \forall \xi>0 .
$$

This condition means that most of the probability mass of $S_{k}$ is in an interval about the mean and this interval is small relative to $w_{n}$. The Lindeberg-Feller Central Limit Theorem states that the Lindeberg-Feller condition implies $W_{n} / w_{n}$ converges in distribution to $N(0,1)$ as $n \rightarrow \infty$, where $N(0,1)$ denotes the standard Gaussian random variable [15, Sec. 9.8].

Recall the ICA representation in Eq. 1, i.e., $\tilde{\mathbf{X}}=\mathbf{A S}$, where $\mathbf{S}$ is a $n$-dimensional random vector with independent components $\left\{S_{k}\right\}$ of mean zero and finite variances, $\tilde{\mathbf{X}}$ is a $\mathbb{R}^{m}$-valued random vector, and $\mathbf{A}$ is the $m \times n$ transformation matrix with components $\left\{A_{j k}\right\}$. Note that we do not restrict $\tilde{\mathbf{X}}$ and $\mathbf{S}$ to have the same dimension, i.e., $m=n$, in this proof. Set

$$
W_{n}=\sum_{j=1}^{m} \beta_{j} \tilde{X}_{j}=\sum_{j=1}^{m} \sum_{k=1}^{n} A_{j k} \beta_{j} S_{k}=\sum_{k=1}^{n}\left[\sum_{j=1}^{m} A_{j k} \beta_{j}\right] S_{k}=\sum_{k=1}^{n} Y_{k}
$$

where $Y_{k}=\left[\sum_{j=1}^{m} A_{j k} \beta_{j}\right] S_{k}$ and $\left\{\beta_{j}\right\}$ are arbitrary constants. The random variables $\left\{Y_{k}\right\}$ have finite variances provided $E\left[S_{k}^{2}\right]<\infty$. Since $\left\{S_{k}\right\}$ are independent and the distributions are assumed to satisfy the Lindeberg-Feller condition, so are $\left\{Y_{k}\right\}$. Accordingly, $W_{n} / w_{n} \rightarrow N(0,1)$ in distribution as $n \rightarrow \infty$ with the notation $w_{n}=\sum_{k=1}^{n} E\left[Y_{k}^{2}\right]$, and since $\left\{\beta_{j}\right\}$ are arbitrary, $\tilde{\mathbf{X}}$ becomes a Gaussian vector as $n$ increases indefinitely. Denote the characteristic functions of $W_{n}$ and $\tilde{\mathbf{X}}$ by $\varphi_{W}(t)$ and $\varphi_{\tilde{X}}(\mathbf{v})$, respectively. The above statement can be proved based on (1) the equivalence of $\varphi_{W}(t)$ 
and $\varphi_{\tilde{X}}(\mathbf{v})$, i.e.,

$$
\varphi_{W}(t)=E\left[\exp \left(i W_{n} t\right)\right]=E\left[\exp \left\{i\left(\sum_{j=1}^{m} \beta_{j} \tilde{X}_{j}\right) t\right\}\right]=E\left[\exp \left\{i\left(\sum_{j=1}^{m} v_{j} \tilde{X}_{j}\right)\right\}\right]=\varphi_{\tilde{X}}(\mathbf{v})
$$

with the notation $v_{j}=\beta_{j} t$, and (2) the fact that $\varphi_{W}(t)$ converges to Gaussian characteristic function as $n \rightarrow \infty$. Hence, $\tilde{\mathbf{X}}$ is approximately Gaussian for sufficiently large $n$.


Figure 9: Marginal distributions of $\mathbf{X}, \tilde{\mathbf{X}}$, and multivariate Gaussian distribution with dimensions: $n=20($ a) and $n=40(\mathrm{~b})$

The above theoretical arguments can be illustrated by a numerical example. Consider a Tdistributed random vector with five degrees-of-freedom. Fig. 9 shows the marginal distributions of $\mathbf{X}, \tilde{\mathbf{X}}$, and multivariate Gaussian distribution with dimensions $n=20$ and $n=40$. It can be seen that the distribution of $\tilde{\mathbf{X}}$ tends to be Gaussian for large $n$.

Generally, the dimension of $\mathbf{X}$ is larger than that of $\mathbf{S}$, i.e., $n<m$. Yet, if the ratio of these dimensions is kept constant, we note that $n \rightarrow \infty$ as $m \rightarrow \infty$ so that the previous statement applies.

\section{Conclusions}

This paper has provided a critical evaluation of the performance of the independent component

analysis (ICA). In ICA, random vectors $\mathbf{X}$ are characterized by vectors $\tilde{\mathbf{X}}$ which are defined by the summations of deterministic vectors with the same dimension, multiplied by independent random variables. We have shown that (1) $\tilde{\mathbf{X}}$ and $\mathbf{X}$ have the same distribution if $\mathbf{X}$ follows Gaussian and non-Gaussian probability laws of which the characteristic functions satisfy some conditions; and 
(2) $\tilde{\mathbf{X}}$ can represent $\mathbf{X}$ in tails if $\mathbf{X}$ is non-Gaussian with independent tails.

However, the representation $\tilde{\mathbf{X}}$ does not, generally, hold for non-Gaussian $\mathbf{X}$. The validity of this statement and the accuracy of $\tilde{\mathbf{X}}$ have been illustrated by several numerical examples for low dimensional non-Gaussian vectors. Moreover, Lindeberg-Feller Central Limit Theorem shows that

$\tilde{\mathbf{X}}$ is approximately Gaussian for high dimensional non-Gaussian vectors. It was concluded that ICA is an useful tool which provides exact representation or satisfactory approximation for some cases, but it needs to be used with great caution for the others.

\section{Acknowledgement}

The work reported in this paper has been supported by the National Science Foundation under Grant CMMI-1265511. The support is gratefully acknowledged.

\section{References}

[1] H. Ahn, E. Choi, and I. Han. Extracting underlying meaningful fratures and canceling noise using independent component analysis for direct marketing. Expert Systems with Applications, 33(1):181-191, 2007.

[2] X. R. Cao and R. W. Liu. General approach to blind source separation. Signal Processing, 44(3):562-571, 1996.

[3] L. Carassale. Analysis of aerodynamic pressure measurements by dynamic coherent structures. Probabilistic Engineering Mechanics, 28:66-74, 2012.

[4] P. Comon. Separation of stochastic processes. Workshop on Higher-Order Spectral Analysis, pages $174-179,1989$.

[5] P. Comon. Independent component analysis, a new concept? Signal Processing, 36(3):287-314, 1994.

[6] B. A. Draper, K. Baek, M. S. Bartlett, and J. R. Beveridge. Recognizing faces with PCA and ICA. Computer Vision and Image Understanding, 91(1-2):115-137, 2003. 
[7] T. S. Ferguson. A representation of the symmetric bivariate cauchy distribution. The Annals of Mathematical Statistics, 33(4):1256-1266, 1962.

[8] M. Grigoriu. Applied Non-Gaussian Processes: Examples, Theory, Simulation, Linear Random Vibration, and MATLAB Solutions. PTR Prentice Hall, Englewood Cliffs, NJ, USA, 1995.

[9] M. Grigoriu. Stochastic calculus: Applications in science and engineering. Birkhäuser, Boston, 2002 .

[10] Joe. H. Multivariate Models and Dependence Concepts. Chapman and Hall, 1997.

[11] A. Hyvärinen and E. Oja. Independent component analysis: Algorithms and applications. Neural Networks, 13(4-5):411-430, 2000.

[12] S. Kotz, N. Balakrishnan, and N. L. Johnson. Continuous Multivariate Distributions. Volume 1: Models and Applications. John Wiley \& Sons, Inc, New York, 2000.

[13] W. V. Li and Q. M. Shao. A normal comparison inequality and its applications. Probability Theory and Related Fields, 122(4):494-508, 2002.

[14] E. Moreau. A generalization of joint-diagonalization criteria for source separation. Signal Processing, 49(3):530-541, 2001.

[15] S. I. Resnick. A probability path. Birkhäuser, Boston, 1998.

[16] R. Rubinstein. Simulation and the Monte Carlo Method. John Wiley \& Sons, New York, NY, 1981.

[17] A. Yeredor. Blind source separation via the second characteristic function. Signal Processing, 80(5):897-902, 2000. 